\title{
Use of mycophenolate mofetil in steroid-dependent and -resistant nephrotic syndrome
}

Received: 4 September 2002 / Revised: 24 March 2003 / Accepted: 24 March 2003 / Published online: 28 May 2003 (C) IPNA 2003

\begin{abstract}
Cyclosporin (Cs-A) is an effective treatment for difficult cases of nephrotic syndrome (NS), but its use can be complicated by renal toxicity and a high incidence of relapses after withdrawal. We reviewed the charts of 10 Cs-A-dependent patients and 4 patients with steroid-dependent nephrotic syndrome (SDNS) not previously treated with Cs-A therapy. All patients had persistent NS, even after prior treatment with oral cyclophosphamide. Of 10 patients treated with Cs-A, 4 had surveillance renal biopsies consistent with Cs-A toxicity, and 8 of 10 had interstitial fibrosis prior to mycophenolate mofetil (MMF). Patients were treated with MMF, at $1,200 \mathrm{mg} / \mathrm{m}^{2}$ per day, in an attempt to allow weaning of Cs-A and/or steroid therapy, and reduce the frequency of relapses. Overall, a significant decrease in frequency of relapses was noted after initiation of MMF therapy. In addition, 5 patients were weaned off Cs-A by 1-2 years of follow-up. One patient was weaned off Cs-A and MMF, and remained in complete remission. However, the subgroup of patients with frequently relapsing SDNS not treated with Cs-A appeared to have a reduction in the number of relapses while on MMF that did not reach statistical significance. Two patients with intractable steroid-resistant NS continued to relapse repeatedly on MMF and Cs-A therapy. We conclude that in this small, single-center, uncontrolled experience, MMF therapy in patients with Cs-A-dependent NS appears to be effective in reducing Cs-A exposure. In addition, MMF appears to significantly decrease the frequency of relapses in this patient population. Further controlled studies are warranted to better define the potential efficacy and side effects of long-term MMF therapy in this setting.
\end{abstract}

G.-M. Barletta ( ) - W. E. Smoyer · T. E. Bunchman

J. T. Flynn - D. B. Kershaw

University of Michigan Health Sciences Center,

Mott Children's Hospital,

1505 Simpson Road East, F6865/Box 0297, Ann Arbor,

MI 48109-0297, USA

e-mail: gpalombo@umich.edu

Tel.: +1-734-9364210, Fax: +1-734-7636997
Keywords Mycophenolate mofetil · Steroid-dependent nephrotic syndrome $\cdot$ Steroid-resistant nephrotic syndrome $\cdot$ Cyclosporin $\cdot$ Treatment

\section{Introduction}

Patients with steroid-resistant nephrotic syndrome (SRNS) and frequently relapsing steroid-dependent nephrotic syndrome (FR-SDNS) often experience significant morbidity due to complications associated with disease, such as acute renal failure, infection, thrombosis, and progression to end-stage renal disease. There is also a significant risk of toxicity secondary to therapeutic regimens that are used to treat these patients $[1,2,3,4]$. Management of refractory disease requires careful balancing of the side effects associated with therapy with the risks associated with persistent NS. Patients with complicated courses of NS often demonstrate highly variable responses to therapy with immunosuppressive agents, including oral and intravenous corticosteroids, cyclophosphamide, and cyclosporin-A (Cs-A) $[1,2,3$, $4,5]$.

Our center has used Cs-A in patients with SRNS to achieve remission. Many of our SRNS patients who have responded to treatment with Cs-A have been difficult to wean off Cs-A without relapse. To treat these Cs-A-dependent patients, as well as patients with FR-SDNS, our center has utilized a 12-week course of cyclophosphamide (168 mg/kg per course) according to International Study of Kidney Disease in Children (ISKDC) guidelines [5] in an attempt to achieve prolonged Cs-A and steroid-free remission, with limited success (unpublished observations).

In patients that continue to have persistent FR-SDNS or Cs-A-dependent SRNS after receiving a 12-week course of cyclophosphamide, the treatment options may include high-dose pulse steroids or initiation/continuation of Cs-A. Both of these therapies involve significant risk for side effects. Although Cs-A is effective in treating such difficult cases of NS, its use can be complicated 
by long-term renal toxicity, as well as persistent relapses after withdrawal $[1,2,3,4]$.

Mycophenolate mofetil (MMF) is an immunosuppressive agent that has been successfully used for the prevention of acute rejection of renal allografts $[6,7]$. More recently, MMF has been reported to be effective in reducing proteinuria in some patients with resistant and idiopathic membranous nephropathy [8], adult onset immune-mediated glomerular disease [9], and, in a case report, in inducing remission of relapsing NS [10]. In an effort to reduce the potential side effects associated with the treatment of difficult-to-manage post-cyclophosphamide SRNS and FR-SDNS, we have used MMF as a third-line agent.

\section{Patients and methods}

We retrospectively analyzed 14 patients with either SRNS or FRSDNS who had been treated with MMF at our center over the past 4 years. SRNS was defined as failure to respond with either complete or partial remission after an initial course of at least 4-8 weeks of daily steroids (prednisone $2 \mathrm{mg} / \mathrm{kg}$ per day). FRSDNS was defined as complete remission [urinary protein excretion of $<4 \mathrm{mg} / \mathrm{h}$ per $\mathrm{m}^{2}$ or reagent strip (Albustix) with negative or trace protein for at least 3 consecutive days] after an initial 4- to 8 -week course of daily steroids, but relapse (urinary protein excretion of $>40 \mathrm{mg} / \mathrm{h}$ per $\mathrm{m}^{2}$ or reagent strip of $2+$ or more protein for 3 consecutive days, having previously been in remission) immediately after weaning to alternate-day steroid dosing, and demonstration of significant steroid toxicity. Patients were considered to have frequently relapsing NS if they had documented two or more relapses within a 6-month period of initial response, or four or more relapses within any 12 -month period.

All renal biopsies were performed percutaneously under ultrasound guidance by using a 16- or 18-gauge biopsy needle (Bard, Covington, Ga., USA). Biopsies were examined by light microscopy (hematoxylin and eosin and periodic acid-Schiff stains), immunofluorescence microscopy (IF), and electron microscopy (EM). Specimens were examined by renal pathologists. Diagnoses were made using standard histological classifications. Minimal change nephrotic syndrome was defined as the absence of abnormalities or mild histological changes noted on light and IF microscopy, with merely diffuse foot-process fusion on EM. IgM nephropathy was classified as diffuse mesangial proliferation/hypercellularity without segmental glomerular sclerosis noted on light microscopy, with mesangial IgM deposition on IF, and foot-process fusion and mesangial immune deposits observed with EM. Focal segmental glomerular sclerosis was identified by the presence of focal glomerular abnormalities with segmental areas of sclerosis. IF was positive for IgM and C3 deposition, and EM demonstrated mesangial immune deposits and foot-process fusion.

MMF was offered as a treatment option for Cs-A- and/or steroid-dependent patients that had failed previous attempts at withdrawal of Cs-A or steroids. Prior to initiating treatment, signed informed consent for MMF treatment was obtained from the patient and/or parents. Families were informed of the potential risks associated with MMF therapy, and offered the option of alternative management with $\mathrm{Cs}-\mathrm{A}$ or continuation of steroids alone. Data collection for this retrospective study was approved by the Institutional Review Board for Human Subject Research (IRBMED).

MMF was begun at an initial dose of $800 \mathrm{mg} / \mathrm{m}^{2}$ per day. All patients were administered $\mathrm{H}_{2}$ blockers at initiation of therapy in an attempt to prevent gastrointestinal side effects. White blood cell count (WBC) was measured 2 weeks after the initiation of therapy and then at monthly intervals. Dosage of MMF was titrated up to $1,200 \mathrm{mg} / \mathrm{m}^{2}$ per day as WBC allowed (target WBC $>4 \times 106 / \mathrm{mm}^{3}$ ). Patients were administered MMF for approximately
8-12 weeks before attempting to wean steroids or Cs-A over the subsequent 6-12 months.

Numerical data were analyzed using paired $t$-test (two-tailed) using Statview (Abacus Concepts, Berkeley, Calif., USA). Data are expressed as mean \pm standard error $(\mathrm{SE})$, with $P$ value $<0.05$ considered significant.

\section{Results}

Patient characteristics are outlined in Table 1. The patients were diagnosed with NS at an average age of 33.5 months (range 12-62 months). All patients carried the diagnosis of either CsA-dependent SRNS or FR-SDNS (5 patients were diagnosed with SRNS and 9 with FR-SDNS). Each patient had been previously treated with at least one 12-week course of oral cyclophosphamide (dosage $168 \mathrm{mg} / \mathrm{kg}$ per course) - 3 patients had received more than one 12 -week course. Seven patients received cyclophosphamide while simultaneously treated with Cs-A. The remaining patients received cyclophosphamide prior to initiation of treatment with Cs-A.

On initial biopsy, 5 patients were noted to have pathology consistent with MCNS, while 9 carried the diagnosis of IgM nephropathy. Two patients (1 initially diagnosed with MCNS and 1 with IgM nephropathy) had follow-up biopsies consistent with FSGS.

Ten of the patients had been treated with Cs-A (Sandimmune) for an average of 77 months (range 48-113 months) and had failed prior attempts to wean Cs-A. These patients were offered MMF in an attempt to allow weaning of Cs-A. Cyclosporine levels prior to initiation of therapy with MMF had been maintained at a whole-blood level (high-performance liquid chromatography) of approximately $80-100 \mathrm{ng} / \mathrm{ml}$. Of the $10 \mathrm{pa}-$ tients on Cs-A, 4 had surveillance biopsies consistent with Cs-A toxicity. In total, 8 of the 10 patients had interstitial fibrosis attributed to primary disease or evidence of Cs-A toxicity on biopsy prior to initiation of MMF. The remaining 4 children with FR-SDNS that had never received Cs-A were started on MMF after repeated relapses following a 12-week course of cyclophosphamide in an attempt to avoid the use of prolonged steroids or adding Cs-A therapy.

The average age of patients at time of initiation of therapy with MMF was 119 months (range 41190 months). The number of relapses in the 12 months prior to initiation of MMF therapy was compared with the number of relapses during the 12 months after starting MMF. The mean number of relapses in the 12 months preceding MMF therapy was $2.85( \pm 0.4)$, compared with $1.07( \pm 0.3)$ in the 12 months following initiation of MMF treatment $( \pm$ standard error, $P<0.01$, paired $t$-test).

Of the 10 patients that were on Cs-A, 5 were weaned to MMF monotherapy. Patients $1,2,3,5$, and 10 were weaned off Cs-A and steroids by 1-2 years of follow-up. Two patients (1 and 10) have remained relapse free. Two patients (2 and 3) had one relapse each and both responded to a short course of steroids without the need to 


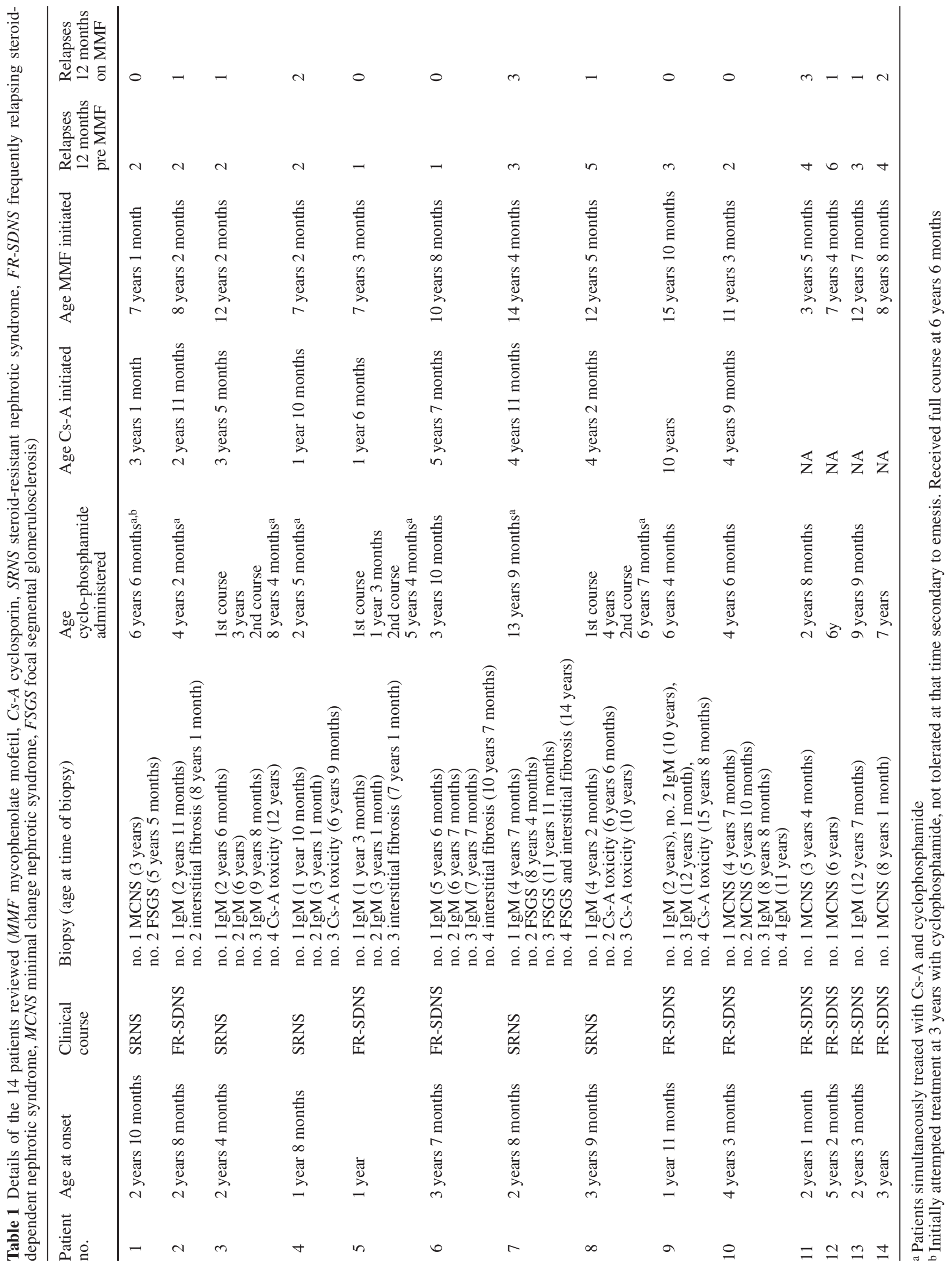


restart Cs-A therapy. In addition, 1 patient (no. 5) was weaned off Cs-A and MMF, and has remained in remission off all therapy for 10 months.

There was a reduction in the dose of Cs-A required to maintain remission in patients 8 and 9. Both were completely weaned off steroids. While tapering Cs-A, patient no. 8 had a relapse treated with a small increase in Cs-A dose, without addition of steroids. Once in remission, Cs-A was weaned again to $25 \%$ of the original (pre MMF) dose, with levels maintained at $40-50 \mathrm{ng} / \mathrm{ml}$, while remaining in remission. Patients 4 and 7, with intractable SRNS, continued to relapse repeatedly on MMF and Cs-A.

The subgroup of patients with FR-SDNS not treated with Cs-A (patients 11-14) appeared to have a slight overall reduction in the number of relapses while on MMF therapy. However, the difference between the number of relapses pre MMF and post initiation of MMF therapy in this subgroup did not reach statistical significance (pre MMF 4.25 \pm 0.63 versus post MMF 1.75 \pm 0.48 , $P=0.06$ ). Nonetheless, there was 1 patient (no. 12) that we were able to wean off steroids and eventually off MMF as well.

Patients 6 and 11 had difficulties tolerating MMF, and experienced significant side effects while on MMF therapy. Patient 6 experienced considerable abdominal discomfort, diarrhea, malaise, and splenomegaly. MMF was therefore discontinued after only 3 months of therapy. This patient remains on Cs-A and steroids, and continues to relapse approximately once a year. Patient 11 had a relatively high rate of relapse even with MMF treatment (3 relapses in 12 months), was off MMF therapy for approximately 6 weeks because of gastrointestinal side effects, and relapsed during that time (this relapse was not included in the analysis). MMF therapy was then resumed, and the patent was able to continue on treatment for the subsequent 12 months. Overall, with close monitoring of WBC and titration of MMF dosages accordingly, no leukopenia $\left(\mathrm{WBC}<2 \times 10^{6} / \mathrm{mm}^{3}\right.$ ) was noted in any of our patients.

\section{Discussion}

Management of persistent NS after treatment with oral cyclophosphamide presents a therapeutic quandary in FR-SDNS and Cs-A-dependent SRNS patients. Treatment options for such refractory disease include Cs-A and high-dose pulse steroids, both of which have significant side effects. Our center has offered MMF to these patients with FR-SDNS and Cs-A-dependent SRNS in an effort to reduce the number of relapses and limit the extent of steroid and Cs-A exposure.

In this retrospective study, there were fewer relapses during the 12 months following initiation of therapy with MMF, than in the 12 months preceding treatment with MMF. Overall, there is a trend toward fewer relapses after initiation of MMF that reaches statistical significance. Many of the relapses that had occurred during
MMF treatment resulted when steroids or Cs-A were being weaned. However, when relapses occurred while on MMF therapy, patients appeared to respond to shorter courses of steroids and/or slight increases in Cs-A doses in order to achieve remission, than they had required prior to starting MMF (unpublished observations). Because this is a retrospective review, we were unable to calculate the precise differences in the average steroid dose per patient year. Six of our patients have been steroid and Cs-A free for several months and are currently being weaned from their MMF-2 patients have already been completely weaned off MMF, and have remained relapse free. Whether there will be any long-lasting benefit of MMF treatment remains to be determined.

Despite appropriate dosing and close monitoring of laboratory studies, some side effects associated with MMF were noted. The presence of gastrointestinal side effects, despite the use of prophylactic $\mathrm{H}_{2}$ blockers, required reduction of dosage and discontinuation of treatment for short periods in 2 of our patients. However, no significant leukopenia or infectious complications were observed in any of the patients included in this study.

In this small, single-center, uncontrolled experience, MMF therapy in patients with FR-SDNS and Cs-Adependent SRNS appears to be effective in reducing steroid and Cs-A exposure, and may represent a potentially less toxic alternative to Cs-A. MMF therapy also appears to help maintain patients in remission and has demonstrated a significant decrease in the overall frequency and severity of relapses after initiation of therapy.

Our experience suggests that MMF may be a useful adjunctive agent in the treatment of difficult cases of NS. It is important to note that all of our patients were responsive to either steroids or Cs-A. Thus, our experience does not apply to treatment of patients with completely resistant NS. Controlled, prospective trials are needed to confirm these findings and better define the optimal duration of MMF therapy. In addition, further studies are warranted to determine the potential efficacy and side effects of long-term MMF therapy in this setting.

\section{References}

1. Mendoza SA, Tune BM (1995) Management of the difficult nephrotic patient. Pediatr Clin North Am 42:1459-1468

2. Ingulli E, Singh A, Baqi N, Ahmad H, Moazami S, Tejani A (1995) Aggressive, long-term cyclosporine therapy for steroidresistant focal segmental glomerulosclerosis. J Am Soc Nephrol 5:1820-1825

3. Gregory MJ, Smoyer WE, Sedman A, Kershaw DB, Valentini RP, Johnson K, Bunchman TE (1996) Long-term cyclosporine therapy for pediatric nephrotic syndrome: a clinical and histologic analysis. J Am Soc Nephrol 7:543-549

4. Collaborative Study Group of Sandimmune in Nephrotic Syndrome (1991) Safety and tolerability of Cyclosporin A (Sandimmune) in idiopathic nephrotic syndrome. Clin Nephrol 35:S48-S60 
5. Report of the International Study of Kidney Disease in Children (1974) Prospective, controlled trial of cyclophosphamide therapy in children with nephrotic syndrome. Lancet II:423-427

6. Tricontinental Mycophenolate Mofetil Renal Transplantation Study Group (1996) A blinded, randomized clinical trial of mycophenolate mofetil for the prevention of acute rejection in cadaveric renal transplantation. Transplantation 61:1029-1037

7. Vanrenterghem Y (1997) The use of mycophenolate mofetil (Cellcept) in renal transplantation. Nephron 76:393-399
8. Miller G, Zimmerman R, Radhakrishnan J, Appel G (2000) Use of mycophenolate mofetil in resistant membranous nephropathy. Am J Kidney Dis 36:250-257

9. Briggs WA, Choi MJ, Scheel PJ (1998) Successful treatment of glomerular disease with mycophenolate mofetil. Am J Kidney Dis 31:213-217

10. Chandra M, Susin M, Abitbol C (2000) Remission of relapsing childhood nephrotic syndrome with mycophenolate mofetil. Pediatr Nephrol 14:224-226 